\title{
Editorial
}

\section{Depth of anaesthesia}

The article by Chi et al. in this issue shows that the changes of the electroencephalogram (EEG) produced by a slow infusion of sufentanil for induction of anaesthesia reached a plateau after $2-4 \mu \mathrm{g} \cdot \mathrm{kg}^{-1}$. Continued administration of sufentanil to a total of $10 \mu \mathrm{g} \cdot \mathrm{kg}^{-1}$ produced minimal subsequent changes. Loss of consciousness, defined as loss of responsiveness to verbal commands, appeared to coincide with or shortly precede maximal EEG changes. The timing between loss of consciousness and maximal EEG changes was not studied in detail. The authors conclude that the EEG may be used to grade the "depth of anaesthesia" during induction until consciousness is lost.

What can we learn from this study about the usefulness of the EEG in measuring "depth of anaesthesia"? "Depth of anaesthesia" is not a unitary concept ${ }^{1}$ and is difficult to define. ${ }^{2}$ There are three dominant themes in the literature. Articles which deal with the "level of depth of anaesthesia" may:

1 demonstrate a relationship between a measurement and the dose or concentration of the anaesthetic agent. An example is the work of Guedel, ${ }^{3}$ who showed that diethyl ether produced a sequence of dose-dependent somatic and autonomic changes, which helped to identify different clinical levels of anaesthesia. Other examples are studies which evaluated changes of the EEG or of sensory-evoked potentials produced by a single anaesthetic agent; ${ }^{2}$

2 show a relationship between a measurement and the level of consciousness. ${ }^{4}$ The problem of unintentional intraoperative awareness or consciousness ${ }^{5}$ is the main source of interest in this question. Studies exploring the possibility of sensory perception and memory in unconscious, adequately anaesthetized ${ }^{6}$ patients may be included as a sub-group in this category;

3 show a relationship between a measurement and the "adequacy" of anaesthesia (usually lack of a somatic or of an adverse haemodynamic response to surgical stimulation). ${ }^{7}$ This is a most difficult task. Haemodynamic response to anaesthesia and surgery is influenced by the direct effects of the anaesthetic agent on: (1) the cardiovascular system (i.e., decreased myocardial con-

From the Departments of Anaesthesia, Royal Victoria Hospital and McGill University, Montreal (Quebec) Canada H3A IA I.
Gilles Plourde MSC MD

tractility); (2) on the spinal cord, where anaesthetic agents interfere with the transmission of nociceptive inputs; ${ }^{8}$ and (3) on the brain (including brain-stem centres for autonomic reflex control of circulation). Other drugs (i.e., beta-adrenergic blockers) also influence haemodynamic responses. It is no surprise that the correlation between haemodynamic responsiveness and the $\mathrm{EEG}^{9}$ or drug concentration ${ }^{10}$ is often poor.

Most of the confusion about the concept of depth of anaesthesia is due to a failure to recognize the differences between these three. Relationships to dose or concentration are the most widely studied in terms of the number of published articles and of positive, reproducible findings. Perhaps because of that success, these studies are sometimes applied to other questions without prior validation. There is no evidence that measurements which show dose or concentration relationships are adequate for studying changes in the level of consciousness. Yet, there are numerous instances when such relationships are suggested as a possible solution to the assessment of consciousness.

It is now easier to answer the question about the usefulness of the EEG in measuring the depth of anaesthesia. If our interest is in a concentration- or dose-response relationship, we can conclude that there is a ceiling effect to the EEG changes produced by sufentanil. If our interest concerns the level of consciousness, we can conclude that loss of consciousness appears to coincide or shortly precede maximal EEG changes. These EEG changes may therefore signal the onset of unconsciousness caused by sufentanil. However, this study does not provide enough information for a definitive conclusion. This would require examining the EEG more closely at the moment of loss of consciousness and also during recovery from anaesthesia.

What is the best way of monitoring the level of consciousness in anaesthetized patients? Consciousness is commonly defined as the appearance of being awake, or as the ability to obey simple verbal commands. Unfortunately, muscle relaxants interfere with these assessments of consciousness. A monitor that would indicate whether consciousness was present or absent would be invaluable. Both the EEG and sensory-evoked potentials have been used for that purpose.

\section{The EEG}

Some EEG patterns always indicate unconsciousness: 
burst suppression, isoelectricity, generalized cpileptic activity. However, there are practically no EEG patterns that prove consciousness is present. ${ }^{11,12}$ The EEG patterns seen during most anaesthetics do not provide clear information about the level of consciousness. ${ }^{13}$ The EEG during $1 \%$ halothane anaesthesia shows a dominant frequency of $8-12 \mathrm{~Hz},{ }^{14}$ which is also the dominant frequency seen in relaxed awake subjects. Diffuse, bilateral delta activity of the type seen during high-dose opioid anaesthesia is not an absolute marker for unconsciousness, since it can be seen in conscious patients. ${ }^{15}$ Whether the predominant delta activity caused by opioids (see the article by Chi et al.) reflects unconsciousness in this specific instance is not established. Whether the EEG would be useful to detect the regaining of consciousness in a pharmacologically paralyzed patient is also not known. The EEG changes during emergence from anaesthesia ${ }^{16}$ are, in part, caused by artifacts from increased muscle tone. ${ }^{17}$ Therefore, the EEG has limited usefulness for monitoring the level of consciousness during anesthesia, although, as suggested by the article of Chi et al., it may be helpful in following the loss of consciousness during induction with opioids.

\section{Sensory-evoked potentials}

The auditory middle latency evoked response (MLR), which is attenuated in a dose-dependent manner by most anaesthetic agents, has been evaluated for assessing the level of consciousness. Latency changes can indicate when responsiveness to verbal command is likely to occur during light anaesthesia with $\mathrm{H}_{2} \mathrm{O},{ }^{18}$ but these findings are not always applicable. The MLR was not affected during anaesthesia with high-dose fentanyl ${ }^{19}$ which, regardless of the controversy about its anacsthetic properties, ${ }^{20}$ produces sedation and unresponsiveness.

The $40 \mathrm{~Hz}$ auditory steady-state reponse (ASSR) is an evoked potential which results from the superimposition of individual MLRs. ${ }^{21}$ The ASSR appears to be a useful indicator for the level of consciousness. Its amplitude is reduced by $50-70 \%$ by sleep ${ }^{22}$ and it is abolished by thiopentone or isoflurane anaesthesia. ${ }^{17}$ The reappearance of the ASSR after anaesthesia coincided with the return of responsiveness to verbal commands. The MLR and ASSR share a common weakness, however: both can be recorded in comatose patients. ${ }^{23-25}$ Their presence therefore does not prove that consciousness is preserved. On the other hand, certain alterations of the responses, when caused by drugs, probably indicate unconsciousness while their disappearance likely signals the regaining of consciousness.

Certain evoked potentials can only be seen in conscious subjects. Two will be considered: the P3 (or P300) and the contingent negative variation (CNV). The P3 is a late component of sensory-evoked potentials. It is associated with the detection of rare, unpredictable target stimuli and is relatively independent of the sensory modality. Several investigators have suggested that the $\mathrm{P} 3$ is associated with access to consciousness. ${ }^{11.26}$ The P3 has been evaluated for assessing consciousness in the peri-anaesthetic period. ${ }^{27}$ It disappeared with loss of responsiveness during induction of anaesthesia and did not reappear with the early signs of awakening during emergence, but only 10-15 minutes later, when the subjects were able to perform simple signal detection. Absence of the P3 does not mean that the patient is unconscious, however, since there may be no P3 if the patient is not paying attention to the stimuli. As a monitor for consciousness, the P3 has high specificity but low sensitivity.

The contingent negative variation (CNV) is a slowly increasing negative potential occurring during the interval between two successive stimuli, when the subject notices a contingency or association between two stimuli. ${ }^{28}$ Typically, CNV protocols use a fixed interval between the two stimuli (e.g., a tone followed two seconds later by a flash), and require a response to the second stimulus (e.g., a button-press after the flash). The first stimulus, therefore, constitutes a warning to prepare for the task. The CNV was found to be more robust than the P3 in predicting responsiveness to verbal command during the administration of $40 \% \mathrm{~N}_{2} \mathrm{O} \cdot{ }^{29}$ It has not been evaluated during general anaesthesia.

Electrical measurements from the scalp provide an excellent method for monitoring ongoing sensory and cognitive processes. More research is needed about the relationships between the EEG or evoked potentials and consciousness, defined usually as behavioral responsiveness. These relationships should be examined during both induction and recovery from anaesthesia. Since anaesthetic concentration and consciousness are not always closely linked, ${ }^{30}$ the ideal monitor for consciousness should reliably reflect the level of consciousness irrespective of drug concentrations.

\section{Acknowledgements}

I am indebted to T.W. Picton for his comments on the text and to $S$. Caney for typing the manuscript.

\section{Le niveau d'anesthésie}

L'article de Chi et al. montre que les changements électroencéphalographiques produits par une injection lente de sufentanil atteignent un plateau après 2-4 $\mu \mathrm{g} \cdot \mathrm{kg}^{-1}$. L'administration subséquente de sufentanil 
jusqu'à un total de $10 \mu \mathrm{g} \cdot \mathrm{kg}^{-1}$ n'a produit que de minimes changements. La perte de conscience, définie comme l'incapacité de répondre aux commandes verbales, a semblé coïncider avec, ou précéder brièvement, les changements électroencéphalographiques maximaux. La relation temporelle entre la perte de conscience et les changements électroencéphalographiques n'a cependant pas été étudiée en détail. Les auteurs ont conclu que l'électroencéphalogramme (EEG) peut être utilisé pour mesurer le "niveau d'anesthésie " durant l'induction avant la perte de conscience.

Que peut-on déduire de cette étude à propos de l'utilité de l'EEG pour évaluer le «niveau d'anesthésie »? L'expression "niveau d'anesthésie» ne constitue pas un concept unitaire ${ }^{1}$ et est difficile à définir. ${ }^{2}$ Trois thèmes semblent dominer la littérature. Les articles concernant le «niveau d'anesthésie " peuvent :

1 démontrer une relation entre une mesure et la dose ou la concentration d'un agent anesthésique. Le travail de Guedel $^{3}$ fournit un exemple. Cet auteur a montré que l'éther diéthylique produit une séquence de changements somatiques et autonomiques, et que ces changements varient selon la dose administrée, permettant d'identifier différents «niveaux cliniques" d'anesthésie. D'autres exemples sont fournis par les études qui évaluent l'effet d'agents anesthésiques sur l'EEG ou sur les potentiels évoqués sensoriels; ${ }^{2}$

2 montrer une relation entre une mesure et le niveau de conscience. ${ }^{4}$ Le problème d'éveil ou de conscience per-opératoire non-volontaire constitue le principal motif d'intérêt pour cette question. Dans cette catégorie l'on peut aussi inclure, à titre de sous-groupe, les études qui explorent les phénomènes de perception sensorielle et de mémoire chez le patient inconscient; ${ }^{6}$

3 montrer une relation entre une mesure et la présence d'une anesthésie "adéquate" (absence de réponse somatique ou hémodynamique défavorable lors du stimulus chirurgical). ${ }^{7}$ Ceci est très difficile. Plusieurs facteurs influencent la réponse hémodynamique. Il faut inclure l'effet des agents anesthésiques sur : (1) le système cardio-vasculaire (i.e., diminution de la contractilité myocardique); (2) sur la mœlle épinière où les agents anesthésiques peuvent modifier la transmission des afférences nociceptives $;^{8}$ et (3) sur le cerveau (incluant les centres pour le contrôle réflexe de la circulation). De plus, d'autres médicaments, tels les bloqueurs beta-adrénergiques, peuvent aussi influencer l'état hémodynamique. Il n'est donc pas surprenant que l'EEG $^{9}$ ou la concentration de l'agent anesthésique ${ }^{10}$ ne puisse pas prédire la réponse hémodynamique.

L'ambiguité qui entoure le concept de niveau d'anesthésie résulte en grande partie du manque de distinction explicite entre ces trois thèmes. Les études portant sur les relations de dose ou de concentration constituent la majorité des articles et des observations concluantes. Peut-être à cause de ce succès, ces études sont souvent utilisées à d'autres fins sans validation préalable. II n'est pas démontré qu'une mesure montrant un effet de dose ou de concentration soit nécessairement adéquate pour étudier le niveau de conscience. Malgré cela, il arrive fréquemment que de telles mesures soient suggérées pour l'évaluation du niveau de conscience.

Il est maintenant plus facilc de répondre à la question de l'utilité de l'EEG pour évaluer le "niveau d'anesthésie ". Si l'on s'intéresse à un effet dose-réponse, l'on peut conclure que les changements électroencéphalographiques produits par le sufentanil plafonnent après $3-4 \mu \mathrm{g} \cdot \mathrm{kg}^{-1}$. Si l'on s'intéresse au niveau de conscience, l'on peut conclure que la perte de conscience coïncide avec, ou précède brièvement, les changements électroencéphalographiques maximaux. Ainsi ces changements peuvent peut-être indiquer que le patient est inconscient. Cependant, l'étude de Chi et al. ne donne pas suffisamment d'information pour une évaluation définitive. Il faudra examiner plus en détail les changements électroencéphalographiques en relation avec le niveau de conscience tant à l'induction de l'anesthésie que lors de l'éveil.

Comment peut-on évaluer le niveau de conscience chez le patient anesthésié ? On évalue normalement le niveau de conscience d'après l'apparence du patient (éveillé ou " endormi ») ou d'après la capacité d'obéir à des ordres simples. Malheureusement, il n'est pas possible d'effectuer cette évaluation en présence de myorelaxants. Il serait donc très utile d'avoir un appareil permettant de déterminer si le patient est conscient ou inconscient. L'EEG et les potentiels évoqués sensoriels peuvent être utilisés à cette fin.

\section{EEG}

Certains tracés électroencéphalographiques sont invariablement associés a un état d'inconscience : isoélectricité, alternance d'isoélectricité et d'activité paroxysmale (burst suppression), activité épileptique généralisée. Cependant, il n'y a pas de tracé électroencéphalographique qui garantisse la présence d'un état de conscience normal. ${ }^{11,12}$ Les tracés électroencéphalographiques observés durant la majorité des anesthésies générales ne donnent pas d'information précise au sujet du niveau de conscience. ${ }^{13}$ L'EEG lors de I'administration d'halothane $(1 \%)$ montre une fréquence dominante de $8-12 \mathrm{~Hz},{ }^{14}$ qui est aussi la fréquence dominante chez le sujet éveillé. L'activité delta bilatérale et généralisée produite par les opiacés n'est pas un marqueur absolu d'inconscience, puisque de tels tracés peuvent être observés chez des patients conscients. ${ }^{15} \mathrm{Il}$ est peut-être possible cependant, comme le suggère l'article de Chi et al., que lors de l'administration d'opiacés, 
l'apparition d'une prédominance delta ${ }^{8}$ reflète l'inconscience. Il n'est pas certain non plus que l'EEG soit utile pour détecter l'éveil non-intentionel en présence de myorelaxants. Les changements électroencéphalographiques observés lors de l'émergence de l'anesthésie ${ }^{16}$ sont, en partie, causés par l'augmentation du tonus musculaire. ${ }^{17}$ En résumé, l'EEG n'a que peu d'utilité pour évaluer le niveau de conscience durant l'anesthésie bien que, comme le suggère Chi et al., l'EEG puisse servir à détecter l'inconscience lors de l'induction avec opiacés.

\section{Potentiels évoqués sensoriels}

La réponse auditive de latence moyenne (RLM), qui est atténuée par la majorité des agents anesthésiques, peut être utilisée pour l'évaluation du niveau de conscience. Il semble que la RLM permette de déterminer la probabilité de réponse à des commandes verbales lors d'une anesthésie légère avec $\mathrm{N}_{2} \mathrm{O}$. Mais ces observations ne s'appliquent pas toujours. La RLM demeure inchangée lors de l'anesthésie avec fentanyl, ${ }^{19}$ qui indépendamment de la controverse au sujet de son effet anesthésique, ${ }^{20}$ produit un état de coma.

Le potentiel évoqué auditif continu de $40 \mathrm{~Hz}$ (PEAC) résulte de la superposition des RLMs individuelles. ${ }^{21}$ Le PEAC peut être un indicateur utile du niveau de conscience. Son amplitude est réduite de 50-70\% lors du sommeil. ${ }^{22}$ Le PEAC est aboli lors de l'anesthésie avec thiopental ou isoflurane. ${ }^{17} \mathrm{La}$ réapparition du PEAC semble coincider avec le retour de la capacité de répondre aux ordres simples. ${ }^{17}$ Le RLM et le PEAC partagent une faiblesse commune cependant : ils peuvent être enregistrés chez des patients comateux. ${ }^{23-25}$ Leur présence, par conséquent, ne prouve pas que le patient est conscient. Toutefois, certaines altérations de ces potentiels évoqués lors de l'anesthésie indiquent probablement l'inconscience, et la disparition de ces altérations après l'anesthésie indique probablement le retour de la conscience.

Certains potentiels évoqués n'apparaissent que chez des patients conscients. Deux seront discutés : l'onde P3 (ou P300), et la variation contingente négative (VCN) (contingent négative variation). L'onde $\mathrm{P} 3$ est une composante tardive des potentiels évoqués sensoriels. Elle survient lors de la détection de stimuli cibles rares et imprévisibles et dépend relativement peu de la modalité sensorielle. Plusieurs chercheurs pensent que l'onde P3 reflète l'accès à la conscience. ${ }^{11,26}$ L'onde $P 3$ peut être utilisée pour évaluer le niveau de conscience durant la période péri-anesthésique. ${ }^{27}$ La P3 disparaît avec la perte de conscience lors de l'induction de l'anesthésie. Elle ne réapparait pas avec les premiers signes d'éveil lors de l'émergence, mais seulement 10-15 minutes plus tard, lorsque les sujets sont capables d'exécutés des tâches de détection de signaux. L'absence de la P3 cependant n'indique pas que le patient est inconscient, puisqu'il n'y aura pas de P3 si le patient ne porte pas attention au stimuli. Comme moniteur de la conscience, par conséquent, la $\mathrm{P} 3$ est très spécifique, mais peu sensible.

La variation contingente négative (VCN) est un potentiel négatif lent qui survient dans l'intervalle entre deux stimuli successifs, lorsque le sujet perçoit une association ou une contingence entre les deux stimuli. ${ }^{28}$ On utilise habituellement un intervalle fixe entre les deux stimuli (par exemple, un son suivi après deux secondes d'un stimulus lumineux) et l'on requiert du sujet une réponse au deuxième stimulus (par exemple, presser un bouton après le stimulus lumineux). Le premier stimulus constitue donc un avertissement de se préparer pour la tâche associée au deuxième stimulus. La VCN s'est révélée plus robuste que la $\mathrm{P} 3$ pour prédire la capacité de répondre aux ordres simples lors de l'administration de doses subanesthésiques de $\mathrm{N}_{2} \mathrm{O}{ }^{29} \mathrm{La}$ VCN n'a pas été évaluée durant l'anesthésie générale.

L'enregistrement de l'activité cérébrale à partir du cuir chevelu constitue une excellente méthode pour étudier en temps réel les processus sensoriels et cognitifs. Il faudra poursuivre les recherches sur les relations entre l'EEG ou les potentiels évoqués et la conscience (habituellement définie comme la capacité de répondre à des ordres simples). Ces relations devront être examinées tant lors de l'induction de l'anesthésie que lors de l'éveil. Comme il peut survenir des dissociations entre les concentrations anesthésiques et le niveau de conscien$\mathrm{ce},{ }^{30}$ le moniteur idéal du niveau de conscience se devra de refléter de façon fiable l'état de conscience indépendamment de la concentration des agents anesthésiques.

\section{Remerciements}

Merci à T.W. Picton pour ses commentaires lors de la rédaction et à $\mathrm{S}$. Caney pour la dactylographie.

\section{References}

1 Prys-Roberts $C$. Anaesthesia: a practical or impractical construct? Br J Anaesth 1987; 59: 1341-5.

2 Stanski DR. Monitoring depth of anesthesia. In: Miller RD (Ed.). Anesthesia, 3rd ed. New York; Churchill Livingstone $1990 ; 1001-30$.

3 Guedel $A E$. Inhalation Anesthesia: a fundamental guide. New York: MacMillan 1937.

4 Levy WJ. Power spectrum correlates of changes in consciousness during anesthetic induction with enflurane. Anesthesiology 1986; 64: 688-93.

5 Bitner RL. Awareness during anesthesia. In: Orkin FK, Cooperman LH (Eds.). Complications in Anesthesiology. Toronto: Lippincott 1983; 349-54.

6 Griffiths $D$, Jones JG. Awareness and memory in anaesthetized patients. Br J Anaesth 1990; 65: 603-6. 
7 Rampil IJ, Matteo RS. Changes in EEG spectral edgc frequency correlate with the hemodynamic response to laryngoscopy and intubation. Ancsthesiology 1987; 67: 139-42.

8 Heavner $J E$. Jamming spinal sensory input: effects of anesthetic and analgesic drugs in the spinal cord dorsal horn. Pain 1975; 1: 239-55.

9 White PF, Boyle WA. Relationship between hemodynamic and clectroencephalographic changes during general anesthesia. Anesth Analg 1989; 68: 177-81.

10 Philbin DM, Rosow CE, Schneider RC, Koski G, D'Ambra $M N$. Fentanyl and sufentanil anesthesia revisited: how much is enough? Anesthesiology 1990; 73: 5-11.

11 Donchin $E, M c$ Carthy $G$, Kutas $M$, Ritter $W$. Eventrelated brain potentials in the study of consciousness. In: Davidson RJ, Schwartz GE, Shapiro D (Eds.). Consciousness and Sclf-regulation. Advances in Research and Theory, Vol 3, New York: Plenum Press 1983; 81121.

12 Simons AJR, Boezeman EHJF, Pronk RAF. Automatic EEG monitoring of anaesthesia. In: Jones JG (Ed.). Depth of Anacsthesia, Toronto: Baillière Tindall 1989; 623-47.

13 Marshall M, Longley BP. Stanton WH. Elcctroencephalography in anaesthetic practice. Br J Anaesth 1965; 37 : 845-57.

14 Stockard J, Bickford R. The neurophysiology of anacsthesia. In: Gordon E (Ed.). A Basis and Practice of Neuroanaesthesia. Amsterdam: North Holland Biomedical Press 1981; 1: 3-46.

15 Sharbrough FW. Nonspecific abnormal EEG patterns. In: Nierdermeyer E, Lopes da Silva F (Eds.). Electroencephalography. Basic principles, clinical applications and related fields. Baltimore: Urban and Schwarzenberg 1987; 163-81.

16 Shah NK, Long CW, Bedford RF. "Delta-shift": an EEG sign of awakening during light isoflurane anesthesia. Anesth Analg 1988; 67: S206 (Abstract).

17 Plourde G, Picton TW. Human auditory steady-state response during general anesthesia. Anesth Analg 1990; 71: $460-8$.

18 Thornton C, Barrowcliffe MP, Konieczko KM et al. The auditory evoked response as an indicator of awareness. Br J Anaesth 1989; 63: 113-5.

19 Kileny $P$, Dobson D, Gelfand ET. Middle-latency auditory evoked responses during open-heart surgery with hypothermia. Electroencephalogr Clin Neurophysiol 1983; 55: 268-76.

20 Wong $K C$. Narcotics are not expected to produce unconsciousness and amnesia. Anesth Analg 1983: 62: 625-6.

21 Galambos $R$, Makeig $S$, Talmachoff PJ. A 40-Hz auditory potential recorded from the human scalp. Proc Natl Acad Sci USA 1981; 78: 2643-7.
22 Linden RD, Campbell KB, Hamel G, Picton TW. Human auditory steady state evoked potentials during slcep. Electrophysiological Techniques in Audiology and Otology 1985; 6: 167-74.

23 Firsching R, Luther J, Eidelberg E, Brown WE Jr, Story $J L, B$ oop $F A$. 40- $\mathrm{Hz}$ middle latency auditory evoked response in comatose patients. Electroencephalogr Clin Neurophysiol 1987; 67: 213-6.

24 Ottaviani $F$, Almadori $G$, Calderazzo AB, Frenguelli $A$, Paludetti $G$. Auditory brain-stem (ABRs) and middle latency auditory responses (MLRs) in the prognosis of severcly head-injured patients. Electroencephalogr Clin Neurophysiol 1986; 65: 196-202.

25 Kaga K, Nagai T, Takamori A, Marsh RR. Auditory short, middle, and long latency responses in acutely comatose patients. Laryngoscope 1985; 95: 321-5.

26 Pritchard WS. Psychophysiology of P300. Psychological Bulletin 1981; 89: 506-40.

27 Plourde G, Picton TW, Kellett A. P300 and the level of consciousness during general anaesthesia. Can J Anaesth 1989; 36: S103-4 (Abstract).

28 Rohrbaugh JW, Gaillard AWK. Sensory and motor aspects of the contingent negative variation. In: Gaillard AWK, Ritter W (Eds.). Tutorials in ERP Research: Endogenous Components. Amsterdam: North-Holland Publishing Company 1983; 269-310.

29 Fenwick P, Bushman J, Howard R, Perry I, Gamble $T$. Contingent negative variation and evoked potential amplitude as a function of inspired nitrous oxide concentration. Electroencephalogr Clin Neurophysiol 1979; 47: 473-82.

30 Gin $T, Y a u G$, Chan $K$ et al. Disposition of propofol infusions for Caesarean Section. Can J Anaesth 1991; 38: 31-6. 